\title{
Conditional observability versus self-duality in a schematic model
}

\author{
Miloslav Znojil \\ Ústav jaderné fyziky AV ČR, \\ 25068 Rež, \\ Czech Republic \\ e-mail: znojil@ujf.cas.cz
}

\begin{abstract}
For a 4-dimensional 3-parametric toy Hamiltonian $H(a, b, c)$ we construct the domain $\mathcal{D}$ of couplings in which the eigenvalues $E_{n}$ remain real (i.e., in principle, observable). A relationship is found between the reflection symmetry of the spectrum (i.e., its Dunne's and Shifman's self-duality $E_{j}=-E_{N+1-j}$ at $N=4$ ) and a geometric symmetry of the physical domain $\mathcal{D}$. Simultaneously, both remain unbroken at $a=c$.
\end{abstract}




\section{Introduction}

Reflection symmetry

$$
E_{j}=-E_{N+1-j}, \quad j=1,2, \ldots
$$

between the low- and high-excitation parts of the energy spectra is one of features of all the many-body Hamiltonians which are quadratic in the creation and annihilation operators [1]. In a different physical context of quasi-exactly solvable local potentials, Dunne and Shifman [2] found the same form of symmetry and called it self-duality of the spectrum. Recently, it was rather surprising for us to detect the emergence of the same spectral symmetry (11) also during the study of the strongcoupling version of a family of finite-dimensional phenomenological non-Hermitian (often called $\mathcal{P} \mathcal{T}$-symmetric) chain-model Hamiltonians [3].

The latter study revealed that the additional symmetry (1) appeared in connection with a thorough simplification of the spectra and also with a facilitated feasibility of an algebraic determination of the positions of certain maximal physical couplings. Formally, the latter maximal couplings were defined as vertices of the boundary $\partial \mathcal{D}$ of the domain $\mathcal{D}$ where the free parameters of our models remained physically acceptable, i.e., compatible with the reality and observability of the energies. As long as the domain $\mathcal{D}$ proved bounded, the observability of the related energies can be called "conditional" [4]. In our subsequent deeper study [5] of the same class of models with symmetry (1) we succeeded in a further constructive extension of the previous result and in a clarification of the geometric structure of the horizons $\partial \mathcal{D}$ in non-empty vicinities of all the above-mentioned vertices. In our present brief communication we feel inspired by the latter experience.

Our purpose is to throw new light on the peculiar apparent correspondence between the spectral symmetry (11) and the geometric shape of the boundary $\partial \mathcal{D}$. An explicit description of the latter manifold will be performed here for a specific, modified model where the up-down symmetry (1) will be manifestly broken. We shall see, in essence, that after the violation of symmetry (11) also the mysterious simplicity of the shape of the surface $\partial \mathcal{D}$ gets lost. For the sake of clarity of our argument, we shall only consider the construction of $\partial \mathcal{D}$ for the exactly solvable three-parametric 
matrix Hamiltonian

$$
H=\left(\begin{array}{cccc}
-3 & b & 0 & 0 \\
-b & -1 & a & 0 \\
0 & -a & 1 & c \\
0 & 0 & -c & 3
\end{array}\right)
$$

The problem differs from its two-parametric $b=c$ predecessor of ref. [3] (cf. also a few related marginal remarks in [6]) by a non-vanishing measure $f>0$ of the extent of the asymmetry which enters the upper coupling defined as $b=b(c, f)=\sqrt{c^{2}+f^{2}}$.

We shall not analyze the possibilities of a transition to the higher-dimensional models here. A thorough discussion of such a perspective has already been presented in both our preceding papers on self-dual cases [3, 5]. Basically, we saw there that the key information about the structure of the spectra is already provided by the models of the lowest dimensions. The growth of the matrix dimension appeared to provide merely technical alterations of the overall picture.

On this background, we now intend to pay more attention to the most elementary up-down-asymmetric model (2). We shall apply computer-assisted linear algebra and formulate some of its consequences in sections 2 and 3 , with a brief summary in section 4 .

\section{Physical domain $\mathcal{D}$ (graphical approach)}

Let us start by a terminological remark concerning eq. (2) and all its generalizations with parameters inside $\mathcal{D}$. Its reason is that the accepted denotation of similar models characterized by a non-standard transposition asymmetry differs in different applications. We will prefer calling our $H$ quasi-Hermitian (a term typical for nuclear physics [7]). Still, several authors also use the equivalent names of pseudo-Hermitian $H$ (predominantly in the context of linear algebra and mathematics [8]) or cryptoHermitian $H$ (this nice and most self-explanatory concept appeared recently in the context of gauge models [9]). Last but not least, another, less strict nickname of $\mathcal{P} \mathcal{T}$-symmetric $H$ as coined by Carl Bender [10] became most popular as particularly appealing in field theory. 


\subsection{The allowed range of $A(a, c, f)$.}

Returning now to our particular model let us recollect that its energies coincide with the roots of its secular determinant,

$$
\operatorname{det}(H-E I)=0
$$

where we decided to set $b^{2}=c^{2}+f^{2}$, with a new measure of the up-down asymmetry $f>0$ replacing the original coupling parameter $b$. We may express eq. (33) in its explicit polynomial form

$$
E_{1,2,3,4}^{4}-A(a, c, f) E_{1,2,3,4}^{2}+C(a, c, f)=4 f^{2} E_{1,2,3,4}
$$

where we have $A(a, c, f)=10-a^{2}-2 c^{2}-f^{2}$ while

$$
C(a, c, f)=9+6 c^{2}-9 a^{2}+3 f^{2}+c^{4}+f^{2} c^{2}
$$

or, in compact form,

$$
\begin{gathered}
C(a, c, f)=\left(3+c^{2}\right)^{2}+f^{2}\left(3+c^{2}\right)-9 a^{2}=\left(3+c^{2}+f^{2} / 2\right)^{2}-\left(9 a^{2}+f^{4} / 4\right)= \\
=\left(8-A / 2-a^{2} / 2\right)^{2}-\left(9 a^{2}+f^{4} / 4\right) .
\end{gathered}
$$

We observe that at any positive $f>0$ and negative (or vanishing) $A \leq 0$, the left-

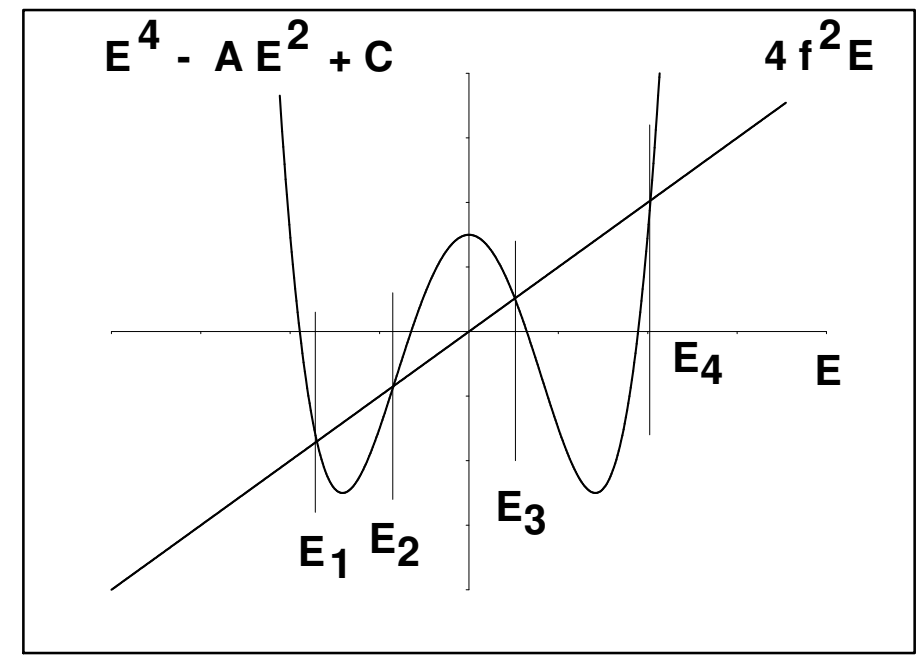

Figure 1: Graphical solution of our secular eq. (4) at $A>0$ ).

hand-side curve of eq. (4) can only have at most two real intersections $E_{1,2}=E_{1,2}(f)$ with the right-hand-side straight line. This means that the spectrum always contains at least two complex conjugate energy roots and we stay off $\mathcal{D}$. 
If we allow $A \leq 0$ and vanishing $f=0$, the two complex roots become real only in the limit $A \rightarrow 0$. Thus, the point of the boundary $\partial \mathcal{D}$ of the physical domain of parameters would be achieved, in full agreement with our older self-dual results [3]. Now, we decided to assume that the self-duality is manifestly broken, $f>0$, so that we may conclude that we have always to consider just the strictly positive values of the polynomial

$$
A(a, c, f)>0, \quad(a, c, f) \in \mathcal{D} .
$$

Under this assumption, the graphical solution of eq. (4) is sampled, in arbitrary units, in Figure 1, At a variable $f>0$ we shall further require that inside $\mathcal{D}$, the left-hand side superposition of the two (viz., quadratic and quartic) parabolic curves of eq. (4) has strictly four real intersections with the right-hand side straight line. From this assumption one immediately deduces that we must have a negative $E_{1}(f)>E_{1}(0)$ and $E_{2}(f)<E_{2}(0)$ and a positive $E_{3}(f)<E_{3}(0)$ and $E_{4}(f)>E_{4}(0)$. Hence, the two rightmost roots $E_{3}$ and $E_{4}$ can never merge and, subsequently, they cannot form a complex conjugate doublet unless $f=A=0$. In terms of the geometry of $\mathcal{D}$, the transition to $f \neq 0$ immediately destroys the multidimensional hedge-hog-shaped form of the surface $\partial \mathcal{D}$ as observed at $f=0$ [5].

\subsection{The allowed range of $C(a, c, f)$.}

An inspection of Figure 1 reveals that at $E=0$, one of the curves acquires the value of $C(a, c, f)$ which may be, in principle, positive, vanishing or negative. Once we keep the quantity $f=f_{0}>0$ fixed, the variation of $a$ and/or $c$ just changes the values of the functions $C\left(a, c, f_{0}\right)$ and $A\left(a, c, f_{0}\right)$. In the two-parametric space of $a$ and $c$ we may move along a line of a constant $A\left(a, c, f_{0}\right)$ and observe that any decrease of $C\left(a, c, f_{0}\right)$ leads to the decrease of the distance between the intersections $E_{2}\left(f_{0}\right)$ and $E_{3}\left(f_{0}\right)$. In principle, these two values will merge and subsequently complexify below certain $f_{0}$-dependent value $C_{(\text {minus })}\left(a, c, f_{0}\right)<0$. Similarly, the growth of the value of the function $C\left(a, c, f_{0}\right)$ will imply the decrease of the distance between the two leftmost energies $E_{1}\left(f_{0}\right)$ and $E_{2}\left(f_{0}\right)$. The latter pair will merge (and subsequently complexify) at an upper bound $C_{(p l u s)}\left(a, c, f_{0}\right)$ which, by the way, need not be necessarily positive. We may conclude that all the four energies remain real iff

$$
C_{(\text {minus })}(a, c, f) \leq C(a, c, f) \leq C_{(\text {plus })}(a, c, f), \quad(a, c, f) \in \mathcal{D} .
$$


Both these bounds follow also algebraically from our secular equation (44) of course. Their occurrence corresponds to the confluence of the above-mentioned pairs of energies at a point of the boundary $\partial \mathcal{D}$ of $\mathcal{D}$.

In the graphical language this means that inside all the interior of $\mathcal{D}$, the graph of the secular polynomial $Y(E)=\operatorname{det}(H-E)=E^{4}-A E^{2}-4 f^{2} E+C$ will intersect the real line four times, achieving its leftmost, negative minimum at a negative real variable $z_{(\min )} \in\left(E_{1}, E_{2}\right)$,

$$
Y\left(z_{(\min )}\right)=z_{(\min )}^{4}-A z_{(\min )}^{2}-4 f^{2} z_{(\min )}+C_{(\text {plus })}<0 .
$$

The subsequent positive maximum of $Y(z)$ will occur at a larger $z_{(\max )} \in\left(E_{2}, E_{3}\right)$,

$$
Y\left(z_{(\max )}\right)=z_{(\max )}^{4}-A z_{(\max )}^{2}-4 f^{2} z_{(\max )}+C_{(\text {minus })}>0 .
$$

As long as we showed above that the two rightmost energy roots $E_{3}$ and $E_{4}$ can never merge, the remaining, third extreme of the function $Y(z)$ at some $z \geq z_{\max }$ may and will be ignored here as redundant.

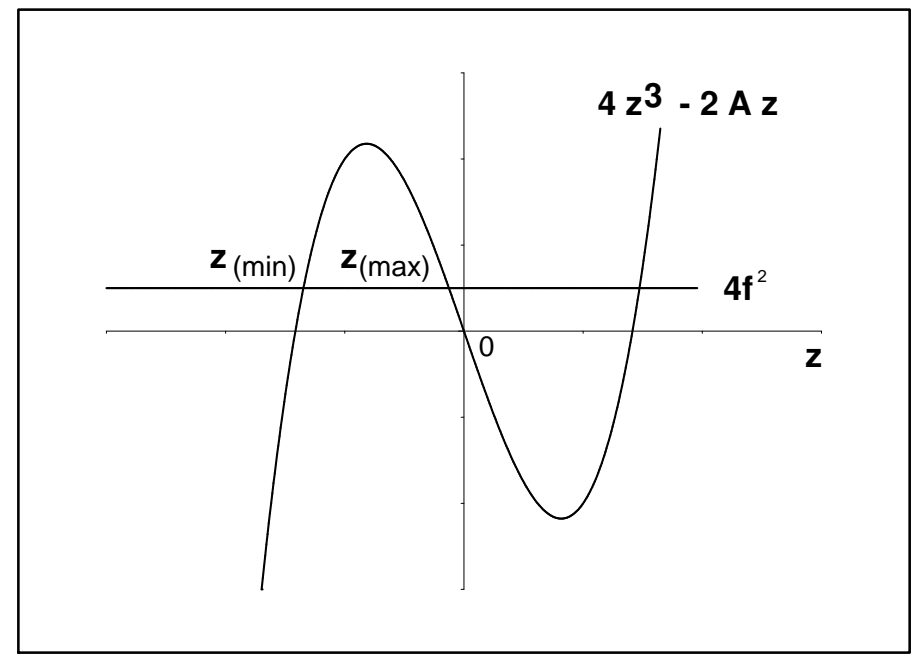

Figure 2: Graphical solution of eq. (9) at $A>0$.

With the variation of $a, c$ and $f$, we shall always have $Y(0) \equiv C$ while the quantities $z_{(\min )} \leq z_{(\max )}$ correspond to the respective leftmost minimum and subsequent maximum of the difference curve $Y(z)$ (in Figure 1, you may imagine that $f$ varies). Algebraically, the two quantities $z_{(\min )}$ and $z_{(\max )}$ coincide with the two real roots of the third-order polynomial $\partial Y / \partial_{z}$, i.e., of the cubic equation

$$
4 z_{\left(\begin{array}{c}
\max \\
\min
\end{array}\right)}^{3}-2 A(a, c, f) z_{\left(\begin{array}{c}
\max \\
\min
\end{array}\right)}=4 f^{2}
$$


(cf. Figure 2). Once we know these auxiliary values, we can define the required bounds from their definition

$$
C_{\left(\begin{array}{c}
\operatorname{minus} \\
\text { plus }
\end{array}\right)}=4 f^{2} z_{\left(\begin{array}{c}
\max \\
\min
\end{array}\right)}+A z_{\left(\begin{array}{l}
\max \\
\min
\end{array}\right)}^{2}-z_{\left(\begin{array}{l}
\max \\
\min
\end{array}\right)}^{4}
$$

which, after an algebraic simplification using eq. (9), degenerates to the simpler formula

$$
C_{\left(\begin{array}{c}
\operatorname{minus} \\
\text { plus }
\end{array}\right)}=\frac{A(a, c, f)}{2} z_{\left(\begin{array}{c}
\max \\
\min
\end{array}\right)}^{2}+3 f^{2} z_{\left(\begin{array}{c}
\max \\
\min
\end{array}\right)} .
$$

We may conclude that since the only negative component of the polynomial $C(a, c, f)$ is $-9 a^{2}$, the latter bounds in effect fix the allowed range of the parameter $a$ for any given pair of $c$ and $f$. In the other words, the middle energy levels $E_{2}$ and $E_{3}$ can merge iff the value of $a$ proves sufficiently large. Moreover, since $A>0$, the quadruple merger of $E_{1}$ and $E_{2}$ and $E_{3}$ with $E_{4}$ would require a return to $f=0$ and proves entirely excluded at $f \neq 0$.

\section{Physical domain $\mathcal{D}$ (analytic approach)}

In our previous studies [3, 5] of $N$-dimensional chain models it has been conjectured and verified that at all $N$, the postulate of a self-duality of the spectrum $E_{j}=-E_{N+1-j}$ could perceivably simplify the construction as well as geometric characterization of the shape of the manifold $\mathcal{D}$. In the preceding section 2 an independent constructive support of the plausibility of such a relationship has been

given via our three-parameter non-selfdual example (2). In what follows we intend to complement these results by paying attention to a simplification and non-numerical description of the boundary $\partial \mathcal{D}$. This might prove relevant, e.g., in the quantitative context of the strong-coupling and/or singular forms of perturbation theory [5] as well as in a more qualitative setting of a possible quantum version of theory of catastrophes [4].

\subsection{The allowed range of the asymmetry $f$.}

We assumed the knowledge of the input energy-degeneracy-determining auxiliary quantities $z_{(\min )}$ and $z_{(\max )}$ which can both be defined by the Cardano formulae in principle. Fortunately, a perceivably less painful recipe can be recommended. In a preparatory step, an inspection of eq. (9) (cf. also Figure 2) reveals that the 
pair of our auxiliary quantities $z_{(\min )} \leq z_{(\max )}$ remains real iff $f \in\left(0, f_{(\text {upper })}\right)$. Here, the numerical value of the upper bound $f_{(\text {upper })}$ annihilates the polynomial $Z(z)=4 z^{3}-2 A z-4 f^{2}$ at its left maximum where $\partial Z / \partial_{z}=0$ at $z=z_{(\text {upper })}$. This means that

$$
12 z_{(\text {upper })}^{2}=2 A, \quad z_{(\text {upper })}=-\sqrt{\frac{A}{6}}, \quad f_{(\text {upper })}=\left(\frac{A^{3}}{54}\right)^{1 / 4} .
$$

Thus, we are allowed to re-parametrize $f \rightarrow \varphi$ in such a way that

$$
f^{2}=f_{(\text {upper })}^{2} \cos \varphi, \quad \varphi=\varphi(A, f) \in(0, \pi / 2) .
$$

Moreover, using the direct insertion in eq. (9) we easily verify that its closed solutions simply read

$$
\begin{aligned}
& z_{(\min )}=-\sqrt{\frac{2 A}{3}} \cos \left(\frac{\pi-\varphi}{3}\right), \\
& z_{(\max )}=-\sqrt{\frac{2 A}{3}} \cos \left(\frac{\varphi+\pi}{3}\right) .
\end{aligned}
$$

Both of them are negative and they only have to satisfy the pair of constraints

$$
\begin{gathered}
z_{(\min )}=z_{(\min )}(A, \varphi) \in(-\sqrt{A / 2},-\sqrt{A / 6}), \\
z_{(\max )}=z_{(\max )}(A, \varphi) \in(-\sqrt{A / 6}, 0) .
\end{gathered}
$$

This enables us to conclude that all the above-mentioned open interval of $\varphi$ may be treated as lying inside $\mathcal{D}$. The angle $\varphi$ is, therefore, a better measure of the breakdown of the self-duality of our toy Hamiltonian $H$. Next, let us show that and why also its further coupling constants should be reparametrized.

\subsection{A reparametrization $(a, c, f) \rightarrow(\alpha, \delta, \varphi)$ of $H$ and $\mathcal{D}$}

Let us replace $A \rightarrow \alpha$ in such a way that $A=10 \sin ^{2} \alpha$ with $\alpha \in(0, \pi / 2)$. Moreover, let us also reparametrize $a \rightarrow \delta$ with $a^{2}=10 \cos ^{2} \alpha \sin ^{2} \delta$ and $\delta \in(0, \pi / 2)$. This implies that the resulting formula $2 c^{2}+f^{2}=10 \cos ^{2} \alpha \cos ^{2} \delta$ may be inserted in the definition of

$$
\begin{gathered}
C(a, c, f)=\left(3+5 \cos ^{2} \alpha \cos ^{2} \delta\right)^{2}-90 \cos ^{2} \alpha \sin ^{2} \delta-f^{4} / 4= \\
=\left(12+5 \cos ^{2} \alpha \cos ^{2} \delta\right)^{2}-90 \cos ^{2} \alpha-135-f^{4} / 4
\end{gathered}
$$


In parallel, we may also reparametrize eq. (11),

$$
C_{\left(\begin{array}{c}
\text { minus } \\
\text { plus }
\end{array}\right)}=\frac{100}{3} \sin ^{4} \alpha \cos \left(\frac{\pi \pm \varphi}{3}\right)\left[\cos \left(\frac{\pi \pm \varphi}{3}\right)-\cos \varphi\right]
$$

which is independent of $\delta$. Thus, we may introduce another pair of shifted and safely positive boundaries

$$
B_{\left(\begin{array}{c}
\text { minus } \\
\text { plus }
\end{array}\right)}=C_{\left(\begin{array}{c}
\text { minus } \\
\text { plus }
\end{array}\right)}+90 \cos ^{2} \alpha+135+f^{4} / 4 \equiv B_{\left(\begin{array}{c}
\text { minus } \\
\text { plus }
\end{array}\right)}(\alpha, \varphi)
$$

and arrive at the final and amazingly compact form of our key constraint ([6),

$$
\sqrt{B_{(\text {minus })}(\alpha, \varphi)} \leq 12+5 \cos ^{2} \alpha \cos ^{2} \delta \leq \sqrt{B_{(\text {plus })}(\alpha, \varphi)}, \quad \delta \in \mathcal{D}
$$

Our construction of the quasi-Hermiticity [7] domain $\mathcal{D}$ is completed since eq. (15) is its definition. In the limit $f \rightarrow 0$, this equation also has been checked to specify the simpler domain $\mathcal{D}$ described in paper 3 .

At $f>0$ our present generalized definition (15) of $\mathcal{D}$ is algebraic and rigorous but its geometric interpretation is not too transparent. Indeed, although our two inequalities specify the allowed, physical range of $\delta$ for any given pair of parameters $(\alpha, \varphi)$, the interval can prove empty. This would mean that the domain $\mathcal{D}$ does not contain any point with pre-selected $(\alpha, \varphi)$. Unfortunately, an account of similar subtleties already lies a bit beyond the scope of our present brief communication.

\section{Summary}

Any non-Hermitian $\mathcal{P} \mathcal{T}$-symmetric quantum Hamiltonian $H$ remains physical in a domain of parameters $\mathcal{D}$ where the spectrum is real and, hence, measurable. In refs. [3, 5] we revealed that several properties of a family of tridiagonal matrix Hamiltonian models of this type become exceptionally transparent after an imposition of the up-down symmetry or "self-duality" requirement (1). In our present study we complemented this observation by a test of the consequences of a manifest violation of the self-duality.

We saw that once the real measure $f^{2}$ of the violation of self-duality becomes different from zero, our first nontrivial, up-down-asymmetric model (2) (skipped in [6] as complicated) offers a challenging eigenvalue problem. We have shown that its discussion and the non-numerical construction of its physical domain of parameters $\mathcal{D}$ still remains feasible and compact. 
We believe that our present construction of a toy model with non-numerically tractable physical horizon $\partial \mathcal{D}$ will further improve our understanding of the mathematics which underlies the so called "conditional-solvability" phenomenon [4] as well as the analyses of the so called "quantum catastrophes" [4, 5, 11]. At present, the practical role of these fairly fresh mathematical concepts finds new and new physical applications, the number of which is rapidly increasing. Besides their above-stressed innovative role in quantum theory and particle physics [10, it is worth noting, in the conclusion, that their use also currently inspired new progress in the areas as remote as relativistic cosmology [8] and phenomenological magnetohydrodynamics [12].

\section{Acknowledgement}

Work supported by GAČR, grant Nr. 202/07/1307.

\section{Figure captions}

Figure 1. Graphical solution of our secular eq. (4) at $A>0$. Figure 2. Graphical solution of eq. (9) at $A>0$. 


\section{References}

[1] J.-P. Blaizot and G. Ripka, Quantum Theory of Finite Systems, MIT, Cambridge, 1986, eq. 3.9 .

[2] G. Dunne and M. Shifman, Annals of Physics 299 (2002) 143.

[3] M. Znojil, J. Phys. A: Math. Theor. 40 (2007) 4863.

[4] M. Znojil, Phys. Lett. B 650 (2007) 440.

[5] M. Znojil, J. Phys. A: Math. Theor. 40 (2007), 13131 (arXiv:0709.1569).

[6] M. Znojil, Phys. Lett. A 367 (2007) 300.

[7] F. G. Scholtz, H. B. Geyer and F. J. W. Hahne, Ann. Phys. (NY) 213 (1992) 74 .

[8] A. Mostafazadeh, J. Math. Phys. 43 (2002) 205 and 2814 and 3944.

[9] A. Smilga, J. Phys. A: Math. Theor. 40 (2007), submitted (arXiv:0706.4064v2 [quant-ph]).

[10] C. M. Bender, Reports on Progress in Physics 70 (2007) 947.

[11] M. Znojil, Phys. Lett. B 647 (2007) 225;

[12] O. Kirillov and U. Günther, J. Phys. A: Math. Gen. 39 (2006) 10057. 